\title{
TFM-PBM simulation model of gas-liquid flow in bubble column with internals
}

\author{
Xiaofeng Guo ${ }^{1,}$, Caixia Chen ${ }^{2, b}$ \\ ${ }^{1,2}$ Key Laboratory of Coal Gasification and Energy Chemical Engineering of Ministry of Education, \\ East China University of Science and Technology, Shanghai, China \\ aguoxiaofeng2010@163.com, bxchen@ecust.edu.cn
}

\begin{abstract}
Keywords: TFM-PBM model; Vertical internals; Bubble coalescence and breakup; Bubble size distribution; Wall lubrication force.

Abstract. An Eulerian two fluid model (TFM) is coupled with the population balance method (PBM) for descriptions of gas-liquid flow in bubble columns with vertical internals. An improved bubble coalescence and breakup model is used in combination of the RNG k- $\varepsilon$ turbulence model and the inter-phase interactions including the drag force and the lift force. A radial wall lubrication force is implemented into the simulation model to take into account the effect of internal walls. The model is validated against literature data in terms of gas holdup and the bubble size distributions. When the internals are present, the turbulent dissipation rates increase significantly in the gaps between the internal walls, and more bubbles with smaller bubble size are predicted in bubble column.
\end{abstract}

\section{Introduction}

Bubble column reactors are important devices for a variety of chemical processes. Many industrial applications, such as liquid methanol synthesis and Fischer-Tropsch (F-T) synthesis, are exothermic reactions. Therefore, internal heat exchangers are used in the bubble columns to remove the excess reaction heat. A thorough understanding of the impacts of internals on the hydrodynamics and the reactor performance is required for a successful design and operation of bubble column reactors.

Computational fluid dynamic simulations of bubble columns are active area of research, however, only empty columns were reported in literatures. Korte ${ }^{[1]}$ studied the heat transfer of horizontal and vertical tube bundles and found that the presence of internals enhanced the heat transfer coefficients of the cooling tubes, partially due to enhancements of bubble breakup rates and the gas-liquid mixing. Chen et al. ${ }^{[2]}$ studied the effect of internals on gas holdup, liquid recirculation, and turbulent parameters in a $0.44 \mathrm{~m}$ diameter bubble column, and concluded that the internals of $5 \%$ of the total column cross-sectional area (CSA) have no significant effect on liquid recirculation velocity. Larachi et al. ${ }^{[3]}$ numerically studied the effect of the CSA and the arrangement of internals on the hydrodynamics of bubble columns. Only the drag force acting on equal sized bubbles was considered for the gas-liquid interactions, and the simulation results were not validated against experimental results.

In this paper, we report a computational model for gas-liquid bubble columns equipped with vertical tube bundles. In the model, the population balance model (PBM) is coupled with two-fluid Eulerian scheme for the gas-liquid fluid dynamics. An improved bubble coalescence and breakup model proposed by the present authors ${ }^{[4]}$ is adopted. A wall lubrication force is introduced to take the fluid-wall interaction into account. The model is benchmarked with a well-documented experiment ${ }^{[5]}$. The simulation results of gas holdup and the bubble size distributions are validated against the data, and the effects of vertical internals on the gas-liquid hydrodynamics are investigated computationally.

\section{Governing equations}

Governing Equations of two-fluid model (TFM)

In the TFM, the liquid is treated as a continuous primary phase and gas is a dispersed secondary phase. The mass and momentum conservation equations are: 


$$
\begin{aligned}
& \frac{\partial\left(\alpha_{i} \rho_{i}\right)}{\partial t}+\nabla \cdot\left(\alpha_{i} \rho_{i} \mathbf{u}_{i}\right)=0 \\
& \frac{\partial\left(\alpha_{i} \rho_{i} \mathbf{u}_{i}\right)}{\partial t}+\nabla \cdot\left(\alpha_{i} \rho_{i} \mathbf{u}_{i} \mathbf{u}_{i}\right)=-\alpha_{i} \nabla p+\nabla \cdot \boldsymbol{\tau}_{i}+\alpha_{i} \rho_{i} \mathbf{g}+\mathbf{F}_{i, j}
\end{aligned}
$$

where the indices i represents the phase. $\mathbf{u}, \alpha, \rho, \tau$ and $\mathbf{F}_{i, j}$ represent velocity vector, volume fraction, density, stress-strain tensor and inter-phase momentum exchange term, respectively.

The RNG $\mathrm{k}-\varepsilon$ is used to describe liquid turbulence, and the turbulence kinetic energy $\mathrm{k}$ and turbulence dissipation rate $\varepsilon$ of liquid phase are computed by:

$$
\begin{aligned}
& \frac{\partial\left(\alpha_{l} \rho_{l} k\right)}{\partial t}+\nabla \cdot\left(\alpha_{l} \rho_{l} \mathbf{u}_{l} k\right)=\nabla \cdot\left(\alpha_{l}\left(\mu_{l}+\rho_{l} C_{\mu} \frac{k^{2}}{\varepsilon \sigma_{k}}\right) \nabla k\right)+\alpha_{l}\left(G_{k, l}+G_{k, g}\right)-\alpha_{l} \rho_{l} \varepsilon+\alpha_{l} \rho_{l} \Pi_{k, l} \\
& \frac{\partial\left(\alpha_{l} \rho_{l} \varepsilon\right)}{\partial t}+\nabla \cdot\left(\alpha_{l} \rho_{l} \mathbf{u}_{l} \varepsilon\right)=\nabla \cdot\left(\alpha_{l}\left(\mu_{l}+\rho_{l} C_{\mu} \frac{k^{2}}{\varepsilon \sigma_{\varepsilon}}\right) \nabla \varepsilon\right)+C_{l \varepsilon}\left(G_{k, l}+C_{3 \varepsilon} G_{k, g}\right) \frac{\alpha_{l} \varepsilon}{k}-C_{2 \varepsilon} \frac{\alpha_{l} \rho_{l} \varepsilon^{2}}{k}+R_{\varepsilon}+\alpha_{l} \rho_{l} \Pi_{\varepsilon, l}
\end{aligned}
$$

where, $C_{1 \varepsilon}, C_{2 \varepsilon}, C_{3 \varepsilon}$ and $C_{\mu}$ are constant, and the default parameter settings are used. $\mu_{l}$ represents liquid viscosity. $\sigma_{k}$ and $\sigma_{\varepsilon}$ are the turbulent Prandtl numbers for $\mathrm{k}$ and $\varepsilon$. $R_{\varepsilon}$ represents the strain rate of the mainstream. $G_{k, l}$ and $G_{k, g}$ represent the generation of turbulent kinetic energy due to the mean velocity gradients and buoyancy. $\Pi_{k, l}$ and $\Pi_{\varepsilon, l}$ are source terms due to the bubble induced turbulence, which is referred to as the eddy effect of bubble trailing vortex. The consumption of bubble momentum caused by drag force was transformed into the turbulent energy of bubble trailing vortex according to Troshko \& Hassan model ${ }^{[6]}$.

The inter-phase momentum closure model

The inter-phase momentum transfer happens in the interface of gas and liquid phases. The drag force reflects the resistance of bubbles when it has a relative velocity to the surrounding liquid:

$$
\mathbf{F}_{l g}^{\text {drag }}=-\mathbf{F}_{g l}^{\text {drag }}=K_{l g}\left(\mathbf{u}_{l}-\mathbf{u}_{g}\right)=\frac{3 C_{d}}{4 d_{b}} \alpha_{g} \rho_{l}\left|\mathbf{u}_{l}-\mathbf{u}_{g}\right|\left(\mathbf{u}_{l}-\mathbf{u}_{g}\right)
$$

where, $F_{\text {lg }}$ denotes the momentum transfer terms from the gas phase to the liquid phase and vice versa for $\mathrm{F}_{\mathrm{gl}} . \quad K_{l g}$ is inter-phase momentum exchange coefficient. $C_{d}$ is drag force coefficient. $d_{b}$ is the bubble diameter. The Ishii-Zuber drag force coefficient model ${ }^{[7]}$ is used.

Due to velocity gradients in radial and azimuth directions, rising bubbles in the liquid are subjected to a lateral lift force. It's correlated to relative velocity and local liquid vorticity:

$$
\mathbf{F}_{l g}^{l i f t}=-\mathbf{F}_{g l}^{l i f t}=-C_{l i j t} \rho_{l} \alpha_{g}\left(\mathbf{u}_{l}-\mathbf{u}_{g}\right) \times\left(\nabla \times \mathbf{u}_{l}\right)
$$

for bubbly flow, Tomiyama et al. ${ }^{[8]}$ observed that the small bubbles tend to move toward the wall while the large ones to the center of the bubble column. The Tomiyama et al.'s model is used for the computation of the lift coefficient $C_{\text {lift }}$.

Due to wall existence, bubbles tend to be pushed away from walls. The wall lubrication force acting on gas bubbles has the general form:

$$
\mathbf{F}_{w g}^{w l}=C_{w l} \rho_{l} \alpha_{g}\left|\left(\boldsymbol{u}_{l}-\boldsymbol{u}_{g}\right)_{\square}\right|^{2} \boldsymbol{n}_{w}
$$

where, $\left|\left(\boldsymbol{u}_{l}-\boldsymbol{u}_{g}\right)_{\square}\right|$ is the phase relative velocity component tangential to the wall surface, $\boldsymbol{n}_{w}$ is the unit normal pointing away from the wall. In the present work, $C_{w l}$ is wall lubrication force coefficient, calculated from Hosokawa et al. model ${ }^{[9]}$.

The population balance model (PBM)

The diameter of bubbles are divided into a number of size bins, and usually, can be classified into two discrete size groups: a smaller size group (bubbles diameters varying from 0.57 to $5.8 \mathrm{~mm}$ ) and a larger size group (bubbles diameters varying from 5.8 to $29.0 \mathrm{~mm}$ ) in accordance with the sign change of lift force. In this work, all bubbles were divided into 18 classes, and the sizes of i-th and (i-1)-th bins bubble classes satisfy $v_{i}=2 v_{i-1}$. The continuity equations are expressed as: 


$$
\frac{\partial \alpha_{\text {small }}}{\partial t}+\nabla \cdot\left(\alpha_{\text {small }} \mathbf{u}_{\text {small }}\right)=\sum_{i=1}^{N_{\text {small }}} S_{i} ; \quad \frac{\partial \alpha_{\text {large }}}{\partial t}+\nabla \cdot\left(\alpha_{\text {large }} \mathbf{u}_{\text {large }}\right)=\sum_{i=1}^{N_{\text {large }}} S_{i}
$$

where, $N$ represents bins of bubble, $S_{i}$ is the source term of i-th bubble group:

$$
S_{i} / V=C_{B, i}-C_{D, i}+B_{B, i}-B_{D, i}
$$

note that, $C_{B, i}, C_{D, i}, B_{B, i}, B_{D, i}$ represent birth rate and death rate due to coalescence and breakup, respectively. The notions of each rates are computed with the Hageseather et al. method ${ }^{[10]}$. The kernel of population balance model is the mechanism of bubble coalescence and breakup, and more details of the improved bubble coalescence and breakup model is referred to our previous work ${ }^{[4]}$.

\section{Validation of the model}

\section{The benchmark cases}

Validation of the above model was preformed based on the experimental setup of Kagumba and Al-Dahhan. A bubble column of $0.14 \mathrm{~m}$ diameter and $1.83 \mathrm{~m}$ height was operated maintaining a constant level of $1.56 \mathrm{~m}$ with superficial gas velocity $0.03 \mathrm{~m} / \mathrm{s}$. Four-point optical probe at axial positions $\mathrm{H} / \mathrm{Dc}=5.1$ was used to measure the bubble sizes and gas holdup.

The numerical simulations were conducted using the ANSYS Fluent 15.0. The QUICK discretization scheme was used for volume fraction and momentum equations, and the second order upwind scheme was specified to discretize other equations. Water and air were used as continuous and dispersed phases, respectively. For all simulations, a velocity inlet was used for the bottom of the column and a pressure outlet was used for the top of the column. No-slip wall boundary conditions were used for liquid phase, and the enhanced near-wall treatment was used for wall-bounded turbulent flows. A time step of $0.01 \mathrm{~s}$ was used and the simulation results were averaged over $80 \mathrm{~s}$ after the flow pattern reached a quasi-steady state. Fig. 1 shows the cross-sectional mesh structures of bubble column used in the simulations. Hexahedral, structured meshes are employed. In the axial direction (z-direction), the grid size is uniformly set to $15.6 \mathrm{~mm}$, and the radial size of the grid is about $4.5 \mathrm{~mm}$.

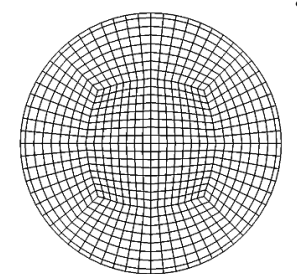

(a) $\mathrm{N}=0$

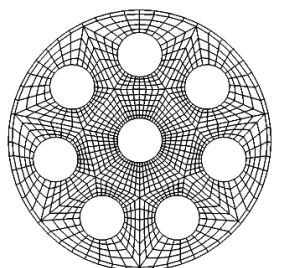

(b) $\mathrm{N}=8$

Fig.1. Computation mesh set-ups.

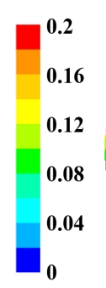

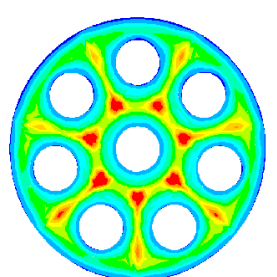

(b) with wlf

Fig.2. Effect of wall lubrication force (wlf) on gas holdup.

\section{Effects of wall lubrication force}

Fig. 2 shows the predicted cross-sectional distributions of time-averaged gas holdup with and without the wall lubrication force (wlf). When wall lubrication force is not included, the gas holdup is much higher near the internals. This is because wall boundary conditions for gas lead to smaller resistance near the wall, and the gas bubbles tend to rise along the solid walls. When the wall lubrication force is included, the gas holdup decreases significantly in the internals region, and much higher in the gaps between the internal walls. This bubble distribution is in line with the experimental results of Chen et al. ${ }^{[2]}$. 


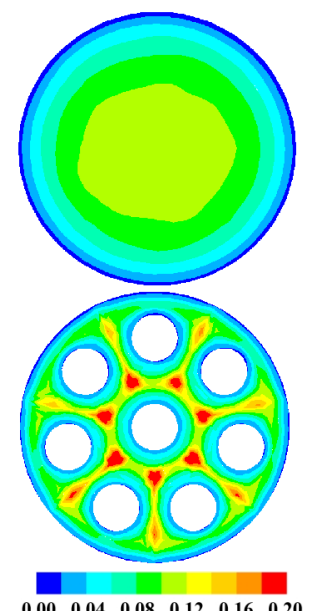

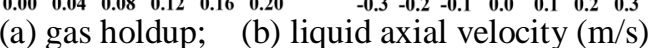

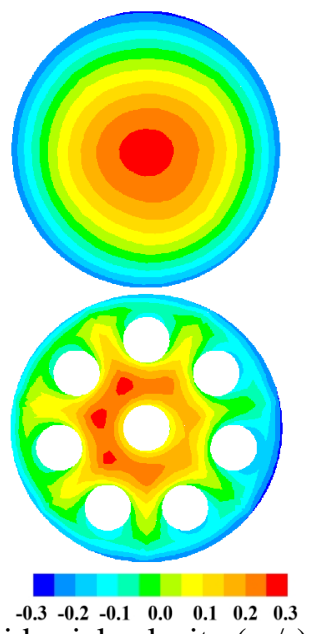

.

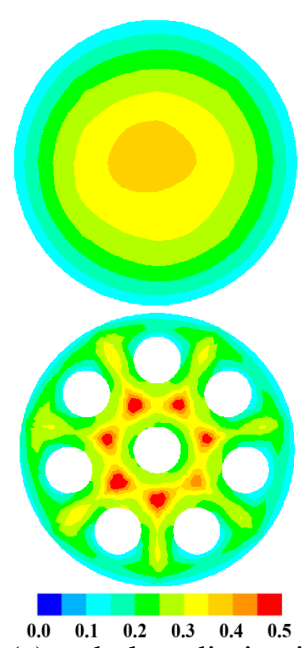

(c) turbulent dissipation rate $\left(\mathrm{m}^{2} / \mathrm{s}^{3}\right)$

Fig.3. Effect of internals on time-averaged flow parameter under superficial gas velocity $0.03 \mathrm{~m} / \mathrm{s}$.

Fig. 3a \& b illustrate the distributions of time-averaged gas holdup and liquid axial velocity at axial position $\mathrm{H} / \mathrm{Dc}=5.1$, respectively. The gas holdup is higher in the center and lower near the wall regions for an empty column. The bubbles tend to segregate at the core region of the column where there is less shear stress than the wall region. When the internals are present, the magnitude of gas holdup changes obviously, especially in the area of $r / R=0.4-0.6$ where the gap between internals is large. The radial distribution of bubbles has been influenced by the internals significantly. Fig. 3c shows time-averaged turbulent dissipation at same axial position. For an empty column, the turbulent dissipation rate is higher in the center region and progressively decreased towards the walls. With internals, the turbulent dissipation rates increase significantly around the central tube.
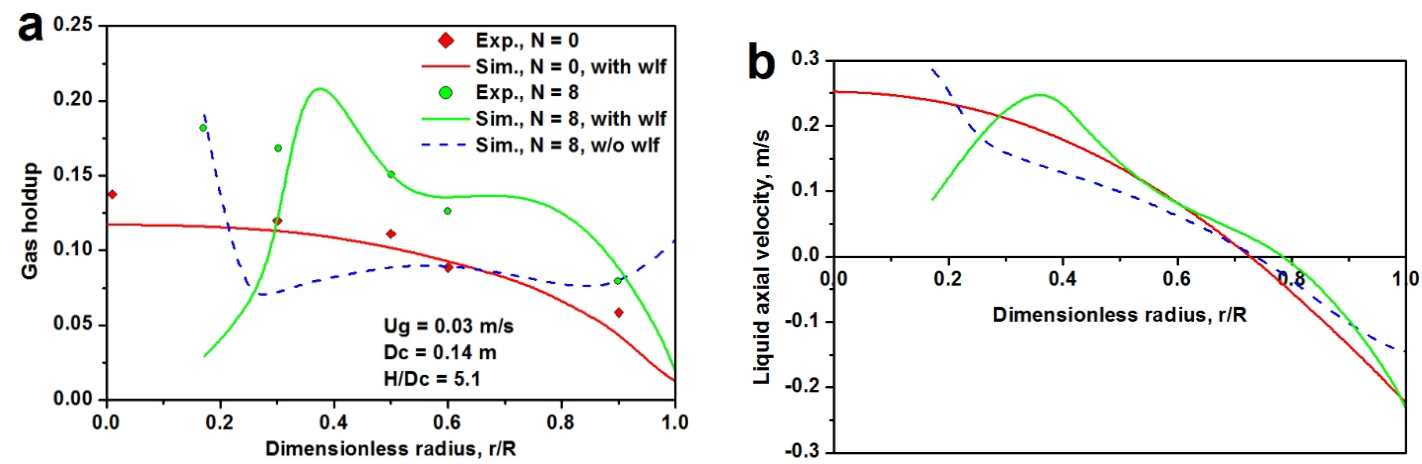

Fig.4. Effect of internals on (a) gas holdup; and (b) liquid axial velocity.

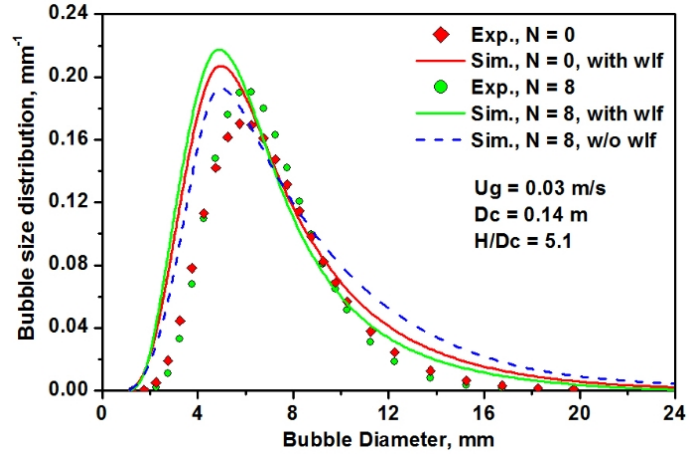

Fig.5. Comparison of predicted bubble size distributions.

The radial profiles of gas holdup and liquid axial velocity drawn in Fig. $4 \mathrm{a} \& \mathrm{~b}$ are compared with the experimental data. Reasonable agreement is obtained for the gas holdups both for the empty column and for the one with internals. Fig. 4b shows that with 8 internal tubes, the liquid velocity reduces near the center. However, the liquid circulation in the region around $r / R=0.4$ is more pronounced. The liquid axial velocity increases slightly with internals, a large circulation still exists, though the peak has been shifted. In agreement with Chen et al., the impact of internals on 
the liquid axial velocity has been limited. Fig. 5 shows the predicted bubble size distributions with and without internals. The log-normal distributions based on the mean and variance bubble size measurements of Kagumba and Al-Dahhan are shown in the same plot for comparison. The bubble size distribution with internals is steeper than that without internals, which is in agreement with the experiment. The reason is that with the presence of internals, an increase in bubble breakup rates results in decreases in the mean bubble sizes. Of note, the bubble coalescence and breakup are primarily influenced by the turbulent kinetic energy dissipation rate of the liquid phase. With turbulent dissipation rate increasing in the gap between internals, bubble breakup frequency increases dramatically, which directly results in a smaller mean bubble size. Smaller bubbles tend to have a longer residence time in the liquid bed, and produce a higher gas holdup in the column. With the influence of the wall lubrication force, fewer bubbles are presented in the internals affecting regions, indicating the wall lubrication force has played a vital role in modeling the gas-liquid flow of a bubble column with internals.

\section{Conclusions}

A TFM-PBM simulation model coupled with an improved bubble coalescence and breakup model is presented. A radial wall lubrication force is implemented to take into account the effects of internal walls. The model is validated against literature data. When the wall lubrication force is included, the predicted distribution of gas holdup increases significantly in the gaps between the internal walls, and the predicted bubble size distributions with and without internals are in good agreements with the log-normal distributions proposed by Kagumba and Al-Dahhan.

\section{Acknowledgement}

This work is supported by China's National Science Foundation (NSFC) under grant 21276085.

\section{References}

[1] H. Korte. Heat transfer in bubble columns with and without internals, Ph.D. Thesis, University of Dortmund, Dortmund, Germany (1987).

[2] J. Chen, et al. Chem. Eng. Sci. 54: 2187-2197 (1999).

[3] F. Larachi, D. Desvigne, L. Donnat, D. Schweich. Chem. Eng. Sci. 61: 4195-4206 (2006).

[4] X. Guo, Q. Zhou, J. Li, C. Chen. Chem. Eng. Sci. 152: 255-266 (2016).

[5] M. Kagumba, M.H. Al-Dahhan. Ind. Eng. Chem. Res. 54: 1359-1372 (2015).

[6] A.A. Troshko, Y.A. Hassan. Int. J. Multiphase Flow 22(11): 1965-2000 (2001).

[7] M. Ishii, N. Zuber. AIChE J. 25: 843-855 (1979).

[8] A. Tomiyama, H. Tamai, I. Zun, S. Hosokawa. Chem. Eng. Sci. 57: 1849-1858 (2002).

[9] S. Hosokawa. Lateral migration of single bubbles due to the presence of wall. In: ASME 2002 Fluids Engineering Division Summer Meeting, Montreal, Quebec, Canada (2002).

[10] L. Hageseather, H.A. Jakobsen, H.F. Svendsen. Chem. Eng. Sci. 57: 3251-3267 (2002). 